\title{
Outcomes of COPD Exacerbations Treated with Corticosteroids, Antibiotics, or Both
}

\author{
Christopher B. Remakus, Francis Cordova, David Ciccolella, \\ A. James Mamary, Matthew R. Lammi, Kartik V. Shenoy, \\ Carla L. Grabianowski, John P. Gaughan, and Gerard J. Criner
}

Division of Pulmonary and Critical Care Medicine, Temple University School of Medicine, 745 Parkinson Pavilion, 3401 North Broad Street, Philadelphia, PA 19140, USA

Correspondence should be addressed to Matthew R. Lammi, matthew.lammi@tuhs.temple.edu

Received 14 June 2011; Accepted 10 July 2011

Academic Editors: A. Michalopoulos and E. Vollmer

Copyright (c) 2011 Christopher B. Remakus et al. This is an open access article distributed under the Creative Commons Attribution License, which permits unrestricted use, distribution, and reproduction in any medium, provided the original work is properly cited.

Background. The outcomes for outpatient treatment of acute exacerbations of COPD (AECOPD) are poorly described. Design. The results of a daily diary recording symptoms and peak flows were compiled into a severity score to trigger algorithm-based treatments and a symptom index to follow treatment response. Treatment failure (symptom index failing to return to baseline for 2 consecutive days or hospitalization within 21 days) was the main outcome. Results. Twenty-two patients $\left(\mathrm{FEV}_{1} 0.81 \pm 0.26 \mathrm{~L}\right)$ were treated for 115 AECOPDs (corticosteroids $=36$, antibiotics $=41$, corticosteroids/antibiotics $=38$ ). Treatment failure was $50 \%$ for the corticosteroid/antibiotic compared to $28 \%(P=0.006)$ for the corticosteroid and $34 \%(P<0.0001)$ for the antibiotic group. Patients suffering from AECOPDs treated with corticosteroids had dyspnea, wheezing, and decreased peak flow; those treated with antibiotics had sputum symptoms; those treated with corticosteroids/antibiotics had dyspnea, wheezing, sputum symptoms, and decreased peak flows. Conclusions. AECOPDs with both dyspnea and sputum symptoms are more refractory to standard treatment and likely require closer monitoring.

\section{Introduction}

Oral corticosteroids and antibiotics, in addition to inhaled bronchodilators, are frequently prescribed for outpatients with acute exacerbations of chronic obstructive pulmonary disease (AECOPDs). The use of corticosteroids in this setting has been shown to improve patients' symptoms and pulmonary function while simultaneously reducing the occurrence of treatment failures $[1,2]$. Likewise, antibiotics have been shown to expedite the recovery in peak expiratory flow while avoiding the occurrence of treatment failures when used as part of an outpatient treatment regimen [3], although the exact indications for their use remain a matter of some conjecture [4].

The Global Initiative for Chronic Obstructive Lung Disease (GOLD) has published guidelines for the use of corticosteroids and antibiotics in the outpatient management of AECOPDs [5]. The outcomes of AECOPDs treatment according to these guidelines, however, have not been well described. The purpose of this study was to characterize the outcomes of AECOPDs treated on an outpatient basis with corticosteroids, antibiotics or a combination of both agents.

\section{Methods}

2.1. Patient Population. A retrospective analysis was performed on data collected from the COPD telemedicine program conducted at our institution, which is used to evaluate daily symptom monitoring using an electronic diary on outcomes in COPD exacerbations. Inclusion and exclusion criteria for the program are listed in Table 1. Prior to inclusion, baseline peak expiratory flow rates and pulmonary function tests were obtained on all subjects. 
TABLE 1: Inclusion and exclusion criteria.

Inclusion criteria

Age 40-80 years

COPD exacerbation requiring hospitalization in 3 months prior to inclusion

Past or current smoking history $\geq 20$ pack-years

$\mathrm{FEV}_{1} \leq 70 \%$

$\mathrm{FEV}_{1} / \mathrm{FVC} \leq 70 \%$

Exclusion criteria

Asthma

Bronchiectasis

Pulmonary fibrosis

Mediastinal or lung mass

Life expectancy $<6$ months

Definition of abbreviations: COPD: chronic obstructive pulmonary disease, $\mathrm{FEV}_{1}$ : forced expiratory volume in 1 second, FVC: forced vital capacity.

Patients were provided with a Palm m500 "LogPad" (Palm Incorporated, Sunnyvale, Calif, USA) electronic diary for recording their symptoms and peak expiratory flow rates on a daily basis. The results were compiled into a severity score (copyright number TX-7-170-236, 2004) used to determine algorithm-based treatments (see Tables 2,3) and a weighted symptom index (copyright \#TX-7-170-236, 2004) used to determine the response to treatment (see Table 4). Patients were provided with an electronic diary and had access to the call center, whose nurses and physicians reviewed the data transmitted from the electronic diaries as well as any calls received from the patients on a daily basis and instituted algorithm-based treatments in accordance with each patient's severity score (see Figure 1).

\section{Definitions}

3.1. COPD Exacerbation. A COPD exacerbation was defined as the initiation of (or increase in the dose of) corticosteroids, the initiation of antibiotics, or the use of both in response to an increase in severity score. Symptoms, peak expiratory flow rate, and change in peak expiratory flow rate from preexacerbation baseline were recorded. For each exacerbation, the symptom index was examined graphically from 7 days before to 21 days after the initiation of treatment (see Figure 2).

3.2. Treatment Success. A treatment success was defined as the return of the Symptom Index to or below the graphically determined baseline for two or more consecutive days within 21 days following the initiation of treatment. Those exacerbations with an initial decrement in symptom index (due to the differences in the calculation of the Severity Score and the Symptom Index), hospitalization (as data was not collected during hospitalizations) or insufficient data (due to patient noncompliance with the electronic diary) were considered nonevaluable for treatment success.
TABLE 2: Components of severity score*.

\begin{tabular}{|c|c|}
\hline Component & Points \\
\hline Increased dyspnea & 1 \\
\hline Increased sputum quantity & 0.5 \\
\hline Purulent sputum ${ }^{\dagger}$ & 0.5 \\
\hline Increased sputum thickness & 0.5 \\
\hline$\geq 2$ minor symptoms & 0.5 \\
\hline \multicolumn{2}{|l|}{ Cough } \\
\hline \multicolumn{2}{|l|}{ Wheezing } \\
\hline \multicolumn{2}{|l|}{ Sore throat } \\
\hline \multicolumn{2}{|l|}{ Nasal congestion } \\
\hline Temperature $>100^{\circ} \mathrm{F}$ & 0.5 \\
\hline Peak expiratory flow rate $\leq 80 \%$ baseline & 1 \\
\hline
\end{tabular}

TABle 3: AECOPD severity score*.

\begin{tabular}{lc}
\hline Severity & Score \\
\hline Mild & $1-1.5$ \\
Moderate & $2-2.5$ \\
Severe & $\geq 3$ \\
\hline
\end{tabular}

${ }^{*}$ Copyright \#TX-7-170-236, 2004.

TABLE 4: Components of symptom index* .

\begin{tabular}{lc}
\hline Component & Points \\
\hline Increased dyspnea $^{\dagger}$ & $0.1-1$ \\
Increased sputum quantity $^{\ddagger}$ & 0.084 \\
Purulent sputum $^{\ddagger}$ & 0.167 \\
Increased sputum thickness $_{\text {Cough }}$ & 0.167 \\
Wheezing & 0.125 \\
Sore throat & 0.125 \\
Nasal congestion & 0.125 \\
Temperature $>100^{\circ} \mathrm{F}$ & 0.125 \\
Peak expiratory flow rate/predicted & $\$$ \\
$>0.6$ & 0.5 \\
$0.5-0.6$ & \\
$0.37-0.49$ & 0.25 \\
$<0.37$ & 0.5 \\
\hline
\end{tabular}

* Copyright \#TX-7-170-236, 2004.

${ }^{\dagger}$ Dyspnea graded from 1-10 and multiplied by 0.1 .

‡Purulent sputum defined as yellow, green or brown colored sputum.

$\S$ Measured peak expiratory flow rate divided by predicted.

3.3. Treatment Failure. The primary outcome was treatment failure, defined as hospitalization or the absence of treatment success in evaluable patients.

3.4. Secondary Outcomes. Secondary outcomes included increased doses of bronchodilators, new or increased doses of corticosteroids, newly prescribed antibiotics, unscheduled primary care visits, emergency room visits, hospitalizations, and the presence of any escalation of care and death. 


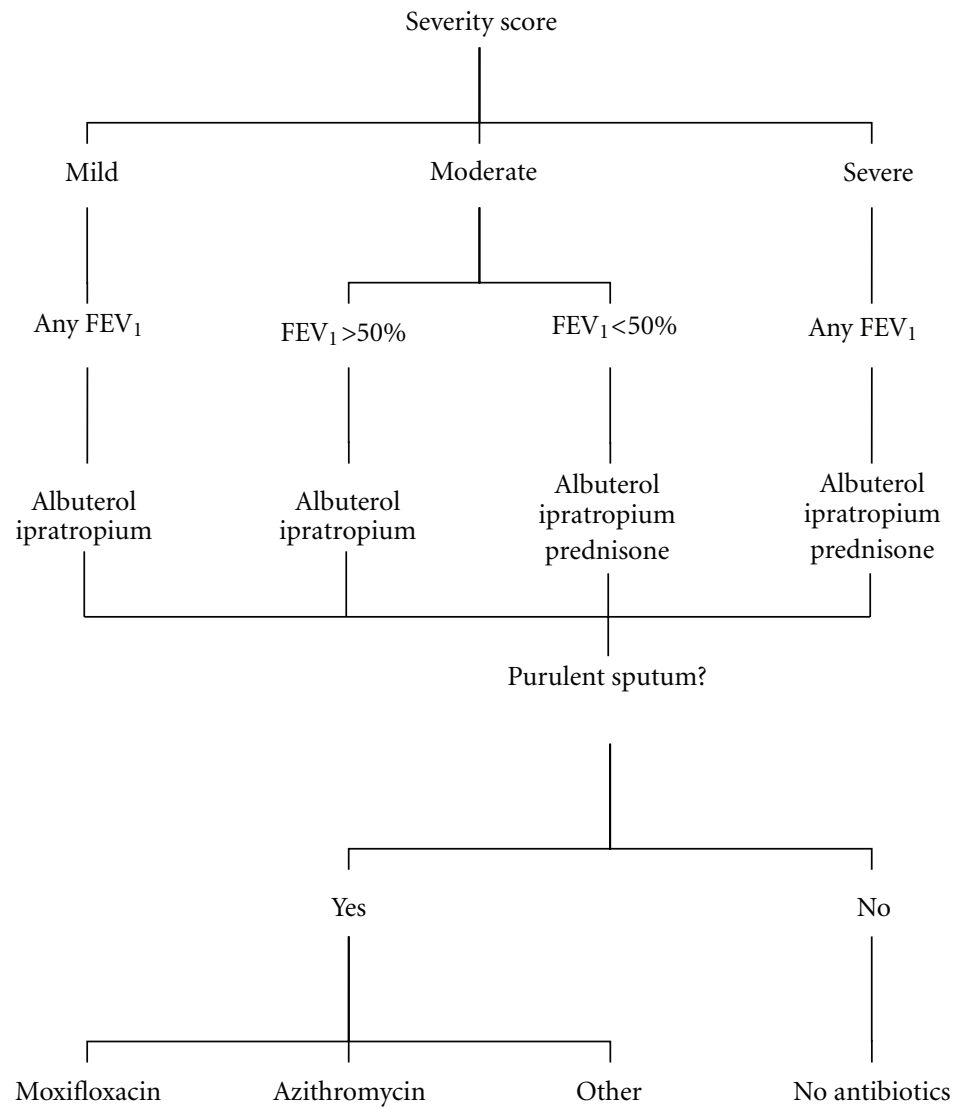

Figure 1: Treatment algorithm for Call Center arm. Prednisone initiated at or increased to $40 \mathrm{mg}$ daily for 10 day course. Moxifloxacin $400 \mathrm{mg}$ daily and azithromycin $500 \mathrm{mg}$ daily preferentially used, but other antibiotics could be prescribed if contraindicated. FEV1: forced expiratory volume in 1 second.

3.5. Data Analysis. For analysis, the exacerbations were grouped according to initial treatment: corticosteroids (C), antibiotics (A), or corticosteroids and antibiotics (CA). The analysis of symptoms, peak flow rates, and symptom Indices used a two-factor repeated measures design (group and time) with included patients evaluated at various time points during the course of therapy. The null hypothesis was that there would be no difference between groups or times. In order to include multiple observations for each patient over time, generalized estimating equations (GEEs) for longitudinal data were used for evaluating exacerbations. GEE is a special case of the generalized linear model taking into consideration the occurrence of events clustered by each individual patient. The correlation structure among measurements from each individual is the basis for using this method. The exacerbation outcomes were assumed to follow a binomial distribution. In this way, the assumption of independence of observations is not violated and is based on the independence of each patient. Since the goal was data mining, followup pairwise comparisons among groups were carried out without adjustment for multiple comparisons [6]. Data analysis was carried out using SAS V9.1.3 software (PROC GENMOD, SAS Institute, Cary, NC, USA).

\section{Results}

4.1. Patient Demographics. Twenty-two patients were treated for 115 AECOPDs. The baseline characteristics of the 22 patients are shown in Table 5. Of the 115 AECOPDs, 36 events $(31 \%)$ were treated with corticosteroids, 41 (36\%) were treated with antibiotics, and 38 (33\%) were treated with both. A breakdown of the type of antibiotics used is shown in Table 6.

4.2. Symptoms Reported during AECOPD. The prevalence of major symptoms reported at the initiation of treatment for the three groups is shown in Figure 3. In addition, the minor symptom cough was reported more commonly in the corticosteroid/antibiotic (92\%) than the corticosteroid group (33\%, $P=0.02$ ), and the minor symptom wheezing was reported more commonly in the corticosteroid (56\%) and the corticosteroid/antibiotic $(66 \%)$ groups than the antibiotic group $(27 \%, P=0.0002$ and 0.001 , resp.), and temperature $>100^{\circ} \mathrm{F}$ was reported more commonly in the corticosteroid/antibiotic (21\%) group than the corticosteroid group $(0 \%, P=0.007)$. There were no other significant differences between the three groups in terms of symptoms. 


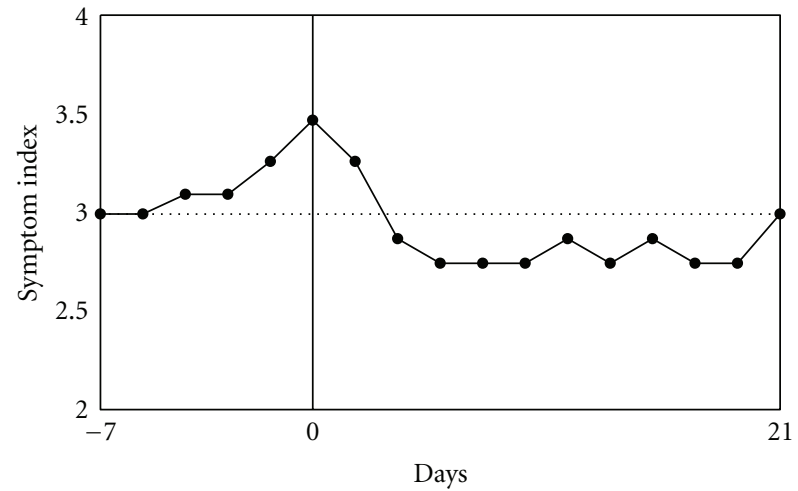

Figure 2: Graph of symptom index for representative exacerbation (Patient \#8). The dashed horizontal line represents the preexacerbation Symptom Index baseline (as determined from the graph); the solid vertical line marks the day when treatment was initiated. In this example, the Symptom Index returns below the preexacerbation baseline on Day \#2 following the initiation of treatment and remains there until Day \#21, indicating a treatment success. (Note that some data points are missing due to patient not reporting on those days.)

TABLE 5: Baseline characteristics of patients treated for AECOPDs $(n=22)$.

\begin{tabular}{lc}
\hline Characteristic & Value \\
\hline Age, years & $65 \pm 6$ \\
Sex & \\
$\quad$ Male & $45 \%$ \\
$\quad$ Female & $55 \%$ \\
Smoking & \\
$\quad$ Active & $10 \%$ \\
Pack-years & $45 \pm 18$ \\
Spirometry & \\
$\quad$ FEV, L & $0.81 \pm 0.26$ \\
$\quad$ FEV, , predicted & $31 \pm 12 \%$ \\
FEV FV $_{1}$ FC & $36 \pm 13 \%$ \\
GOLD stage & \\
I & $0 \%$ \\
II & $9 \%$ \\
III & $32 \%$ \\
IV & $59 \%$ \\
Followup, days & $336 \pm 151$ \\
\hline
\end{tabular}

AECOPDs: acute exacerbations of COPD, $\mathrm{FEV}_{1}$ : forced expiratory volume in 1 second, FVC: forced vital capacity, GOLD: Global Initiative for Chronic Obstructive Lung Disease.

4.3. Peak Flow Rates during AECOPD. There were no significant difference between the peak expiratory flow rates at the initiation of treatment, but there was a significantly greater decrement in the peak expiratory flow rate from baseline in the corticosteroid $(-45.4 \pm 90.1 \mathrm{~L} / \mathrm{s})$ and corticosteroid/antibiotic $(-20.0 \pm 40.7 \mathrm{~L} / \mathrm{s})$ groups than in the antibiotic group $(-3.9 \pm 22.8 \mathrm{~L} / \mathrm{s}, P=0.002$ and 0.04 , resp., see Figure 4).
TABLE 6: Frequency of antibiotics used in the antibiotic (A) and corticosteroid/antibiotic (CA) groups.

\begin{tabular}{lcc}
\hline Antibiotic & $\mathrm{A}(n=41)$ & $\mathrm{CA}(n=38)$ \\
\hline Moxifloxacin & 15 & 10 \\
Azithromycin & 22 & 24 \\
Levofloxacin & 3 & 0 \\
Ciprofloxacin & 1 & 2 \\
Erythromycin & 0 & 1 \\
Trimethoprim/sulfamethoxazole & 0 & 1 \\
\hline
\end{tabular}

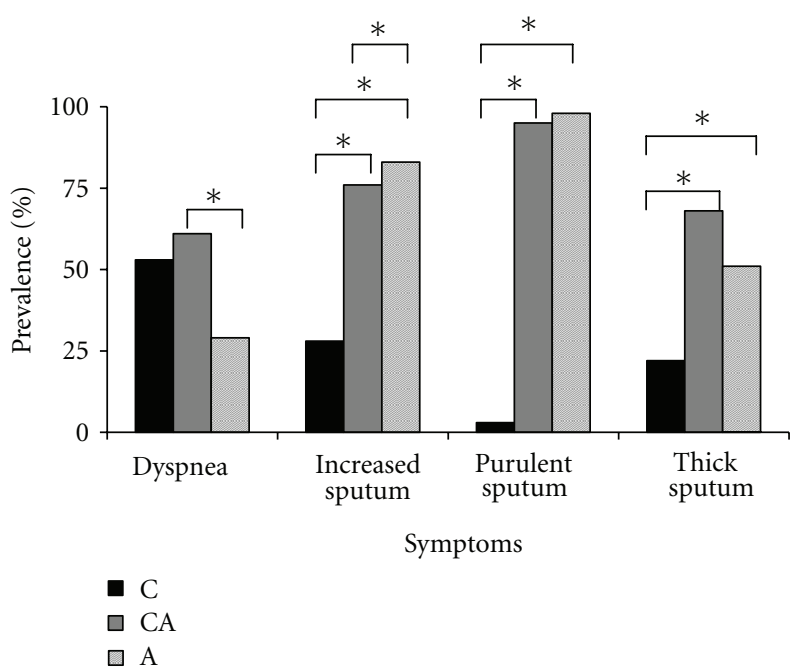

FIgure 3: Prevalence of major symptoms at the initiation of treatment in the corticosteroid (C), antibiotic (A), and corticosteroid/ antibiotic (CA) groups. ${ }^{*} P<0.05$; all others are not significant.

4.4. Symptom Indices and AECOPD. Conversely, there was no significant differences in the changes in the Symptom Index from baseline between the three groups, but the Symptom Index at the initiation of treatment was significantly greater in the antibiotic $(2.08 \pm 0.59)$ and corticosteroid/ antibiotic groups $(2.45 \pm 0.67)$ than the corticosteroid group $(1.43 \pm 0.57, P=0.003$ and 0.002 , resp.).

4.5. AECOPD Treatment Outcomes. Treatment successes among evaluable exacerbations were $21 / 27$ (78\%) in the corticosteroid, 25/36 (69\%) in the antibiotic, and 17/27 (63\%) in the corticosteroid/antibiotic groups, with treatment successes being significantly different between the adjusted antibiotic and corticosteroid/antibiotic group rates $(P=$ 0.01). Among treatment successes, there were no significant differences in the mean time to normalization of the Symptom Index after the initiation of treatment in the corticosteroid ( $5.1 \pm 4.4$ days), antibiotic ( $7.3 \pm 5.4$ days $)$, or corticosteroid/antibiotic groups $(6.9 \pm 4.5$ days $)$. There were 7 hospitalizations in the corticosteroid/antibiotic compared to 2 in the corticosteroid and 2 in the antibiotic groups, but this did not reach statistical significance $(P=0.5$ and 0.3 , resp.). When combining evaluable exacerbations that did not 


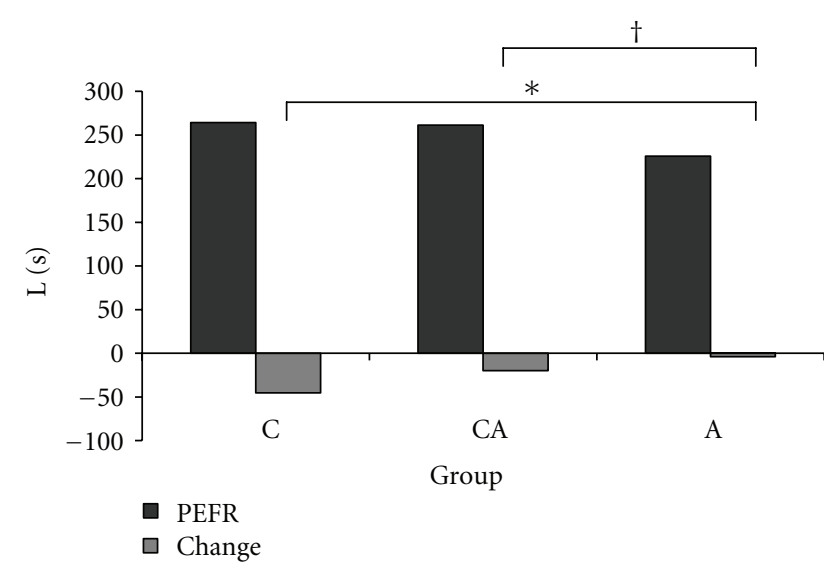

FIGURE 4: Mean peak expiratory flow rates (PEFR) at the initiation of treatment and change from graphically determined preexacerbation baseline in the corticosteroid (C), antibiotic (A) and corticosteroid/antibiotic (CA) groups. ${ }^{*} P=0.002,{ }^{\dagger} P=0.04$, all others not significant.

end in treatment success with exacerbations requiring hospitalization, there was a higher incidence of treatment failure in the corticosteroid/antibiotic group $(17 / 34,50 \%)$ compared to the corticosteroid $(8 / 29,28 \%, P=0.006)$ and antibiotic groups $(13 / 38,34 \%, P<0.0001)$. There were no significant differences in treatment failures for exacerbations treated with moxifloxacin and those treated with azithromycin $(P=$ $0.9)$. Secondary outcomes are shown in Table 7 . There was a higher incidence of exacerbations requiring an escalation of care in the antibiotic (17\%) and corticosteroid/antibiotic groups $(21 \%)$ than the corticosteroid group $(11 \%, P=0.05$ and 0.009 , resp.).

\section{Discussion}

In this paper, we present outcomes for AECOPDs treated on an outpatient basis using published guidelines. Interestingly, AECOPDs treated with both corticosteroids and antibiotics experienced a higher incidence of treatment failure than those treated with corticosteroids or antibiotics alone. These data seem to highlight the importance of specific symptomatology in the prognosis and treatment of COPD exacerbations.

In our study, exacerbations treated with corticosteroids tended to present most prominently with dyspnea, wheezing, and a decrement in peak expiratory flow rate; exacerbations treated with antibiotics tended to present with prominent sputum symptoms. Exacerbations treated with both corticosteroids and antibiotics tended to present with dyspnea, wheezing, sputum symptoms, and a decrement in peak expiratory flow rate. These data suggest that symptoms due to bronchospasm and lower respiratory tract infection can occur independently of each other in any given exacerbation, and that the presence of both portends a more severe exacerbation and a worsened prognosis. Therefore, patients presenting with symptoms of bronchospasm and infection likely require bronchodilators, corticosteroids, and antibiotics and
TABLE 7: Secondary outcomes for corticosteroid (C), antibiotic (A), and corticosteroid/antibiotic (CA) groups.

\begin{tabular}{lccc}
\hline & $\mathrm{C}(n=36)$ & $\mathrm{A}(n=41)$ & $\mathrm{CA}(n=38)$ \\
\hline $\begin{array}{l}\text { Increased bronchodilators } \\
\text { New or increased }\end{array}$ & $1(3 \%)$ & $2(5 \%)$ & $1(3 \%)$ \\
corticosteroids & $2(6 \%)^{*}$ & $4(10 \%)$ & $0(0 \%)^{*}$ \\
New antibiotics & $3(8 \%)$ & $1(2 \%)$ & $0(0 \%)$ \\
$\begin{array}{l}\text { Unscheduled primary } \\
\text { care visit }\end{array}$ & $0(0 \%)$ & $1(2 \%)^{\dagger}$ & $1(3 \%)^{\dagger}$ \\
Emergency room visit & $0(0 \%)$ & $1(2 \%)$ & $5(13 \%)$ \\
Hospitalization & $2(6 \%)$ & $2(5 \%)$ & $7(18 \%)$ \\
Death & $0(0 \%)$ & $1(2 \%)$ & $2(5 \%)$ \\
\hline
\end{tabular}

${ }^{*} P=0.01,{ }^{\dagger} P=0.005$; all others are not significant.

closer followup with a low threshold for hospitalization. Conversely, it seems reasonable to withhold antibiotics in outpatients who do not have clinical evidence of infection at the time of AECOPD presentation and systemic corticosteroids in outpatients with minimal bronchospasm and less severe obstruction at AECOPD presentation, in accordance with current GOLD guidelines.

There was a higher incidence of reported fever (temperature $>100^{\circ} \mathrm{F}$ ) in the corticosteroid/antibiotic group (21\%) compared to the corticosteroid group $(0 \%)$ and the antibiotic group (2\%), although this only reached statistical significance between the corticosteroid and corticosteroid/antibiotic groups. This could suggest that the corticosteroid/antibiotic group had more severe infections than the other two groups, which may have accounted for the differences in treatment outcomes. However, only 3 of the 8 exacerbations in the corticosteroid/antibiotic group in which fever was reported went on to experience treatment failure, so it is unlikely that the higher incidence of treatment failure in this group can be completely attributed to a greater severity of infection as manifested by fever.

The most frequent escalation of care in the corticosteroid group was the addition of antibiotics and the most frequent escalation of care in the antibiotic group was the addition (or increase in chronic dose) of corticosteroids, whereas the most frequent escalation of care in the corticosteroid/antibiotic group was hospitalization. Indeed, some of the exacerbations initially treated with corticosteroids were eventually treated with antibiotics, and some of the exacerbations initially treated with antibiotics were eventually treated with corticosteroids. However, the majority of these exacerbations experienced treatment failure, so the escalations of care during these exacerbations do not explain the better outcomes in the corticosteroid and antibiotic groups. Nevertheless, this seems to underscore the point that any patient on maximal medical therapy for a COPD exacerbation requires close followup to rule out progression of disease and the need for hospitalization.

Telemedical technology is playing an increasing role in medical research and treatment. It seems particularly suited to data acquisition for chronic diseases such as COPD and has already been used for this purpose $[7,8]$. With the help 
of this technology, we were able to monitor COPD patients on a daily basis, recognize and treat acute exacerbations, and monitor the response to treatment. The wealth of data afforded by such technology offers new insights into the natural history of COPD exacerbations, from the prodromal period through the recovery stage, as well as the capabilities and limitations of currently available treatment.

One limitation of our study is that our patient population was small. With data on only 22 patients, it is difficult to make broad generalizations regarding all patients with COPD. In addition, our patient population was heavily skewed towards those with more severe disease. With $91 \%$ of the patients at GOLD stages III or IV, our findings might not be applicable to those with mild to moderate COPD. A larger trial representing patients with all stages of COPD would be required to validate the findings in this study. Lastly, our analysis was retrospective, which inherently limits the strength of conclusions that can be drawn from it.

Nevertheless, we are presenting data on a well-characterized cohort of COPD patients who were monitored for symptoms and peak flows on a daily basis, risk-stratified according to a novel Symptom Index, and treated according to established guidelines. These results have clinical meaning and can easily be transitioned into daily practice.

\section{Conflict of Interests}

None of the authors of the current study have any real or potential conflicts of interest with the present study. Specifically, none of the authors have a direct financial relationship with any commercial entity mentioned in the paper that might lead to a conflict of interest.

\section{Acknowledgments}

The authors acknowledge the grant support of Pennsylvania Study of COPD Exacerbations (PA-SCOPE) PA-DOH 02-7002 .

\section{References}

[1] W. H. Thompson, C. P. Nielson, P. Carvalho, N. B. Charan, and J. J. Crowley, "Controlled trial of oral prednisone in outpatients with acute COPD exacerbation," The American Journal of Respiratory and Critical Care Medicine, vol. 154, no. 2, part 1, pp. 407-412, 1996.

[2] S. D. Aaron, K. L. Vandemheen, P. Hebert et al., "Outpatient oral prednisone after emergency treatment of chronic obstructive pulmonary disease," The New England Journal of Medicine, vol. 348, no. 26, pp. 2618-2625, 2003.

[3] N. R. Anthonisen, J. Manfreda, C. P. Warren, E. S. Hershfield, G. K. Harding, and N. A. Nelson, "Antiobiotic therapy in exacerbations of chronic obstructive pulmonary disease," Annals of Internal Medicine, vol. 106, no. 2, pp. 196-204, 1987.

[4] S. Saint, S. Bent, E. Vittinghoff, and D. Grady, "Antibiotics in chronic obstructive pulmonary disease exacerbations. A metaanalysis," Journal of the American Medical Association, vol. 273, no. 12, pp. 957-960, 1995.

[5] K. F. Rabe, S. Hurd, A. Anzueto et al., "Global strategy for the diagnosis, management, and prevention of chronic obstructive pulmonary disease: GOLD executive summary," The American Journal of Respiratory and Critical Care Medicine, vol. 176, no. 6, pp. 532-555, 2007.

[6] P. J. Diggle, K. Y. Liang, and S. L. Zeger, Analysis of Longitudinal Data, Oxford University Press, New York, NY, USA, 1994.

[7] J. A. Blumenthal, M. A. Babyak, F. J. Keefe et al., "Telephonebased coping skills training for patients awaiting lung transplantation," Journal of Consulting and Clinical Psychology, vol. 74, no. 3, pp. 535-544, 2006.

[8] M. Vitacca, L. Bianchi, A. Guerra et al., "Tele-assistance in chronic respiratory failure patients: a randomised clinical trial," The European Respiratory Journal, vol. 33, no. 2, pp. 411-418, 2009. 


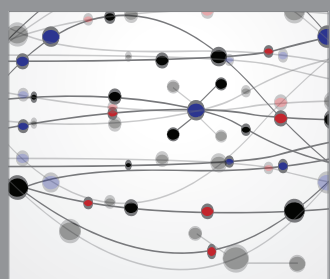

The Scientific World Journal
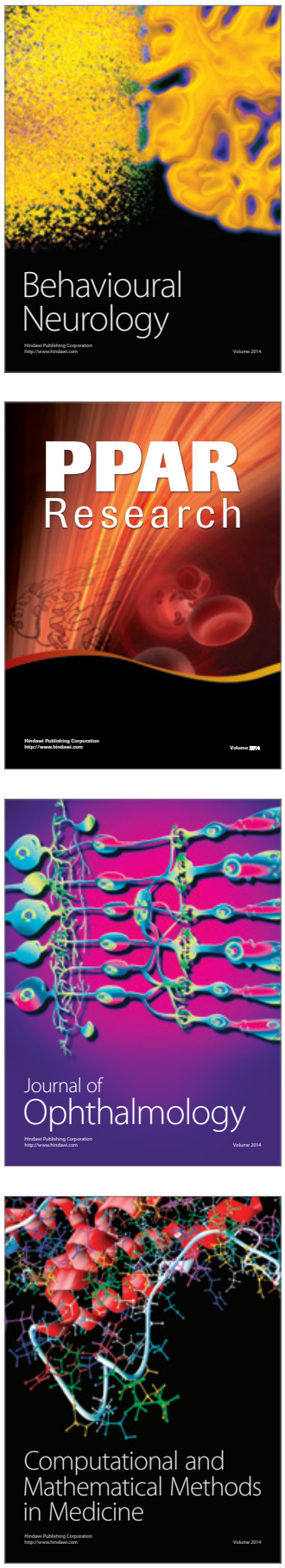

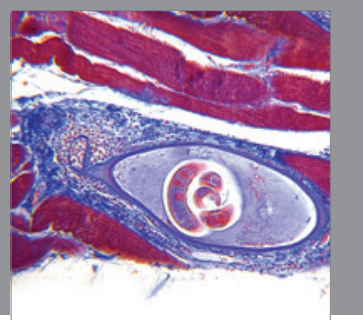

Gastroenterology

Research and Practice
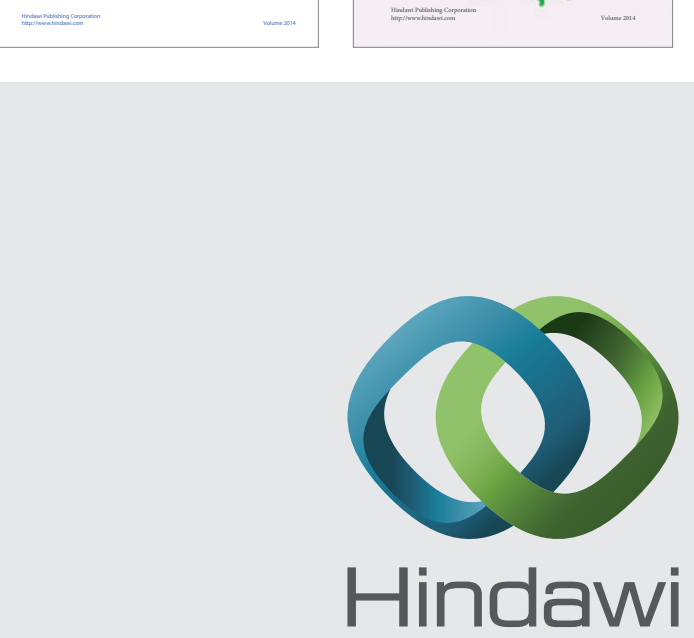

Submit your manuscripts at

http://www.hindawi.com
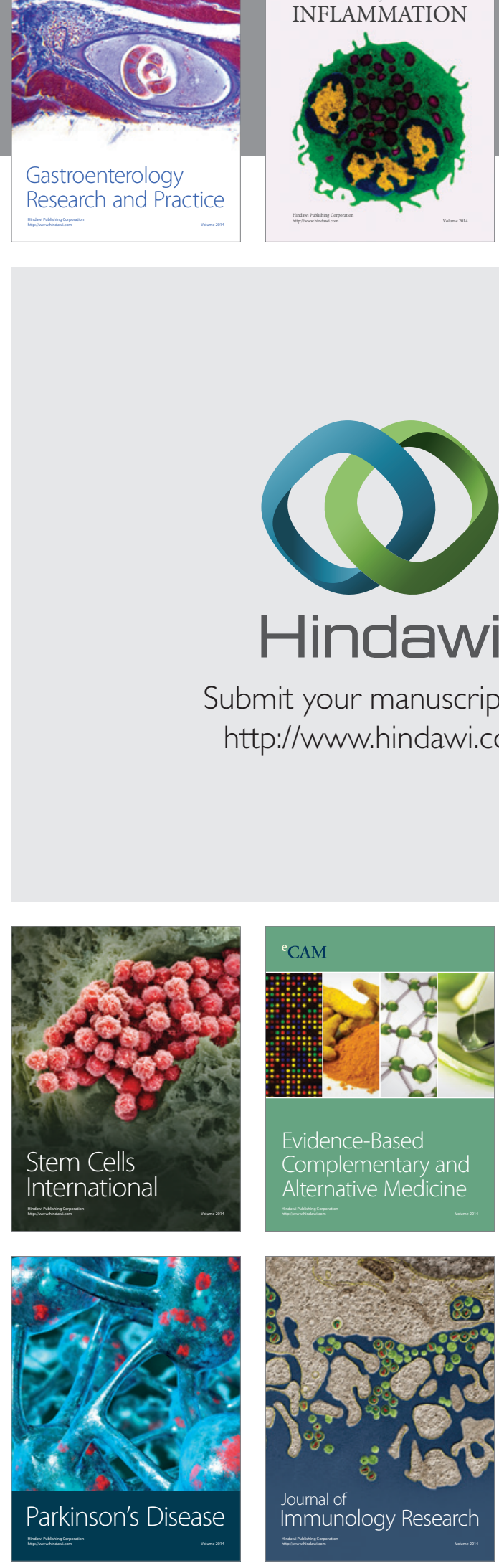

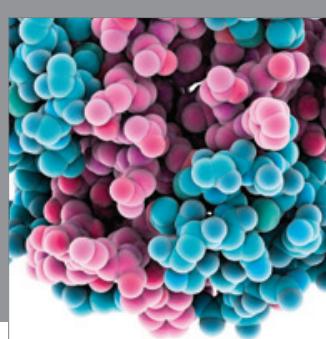

Diabetes Research
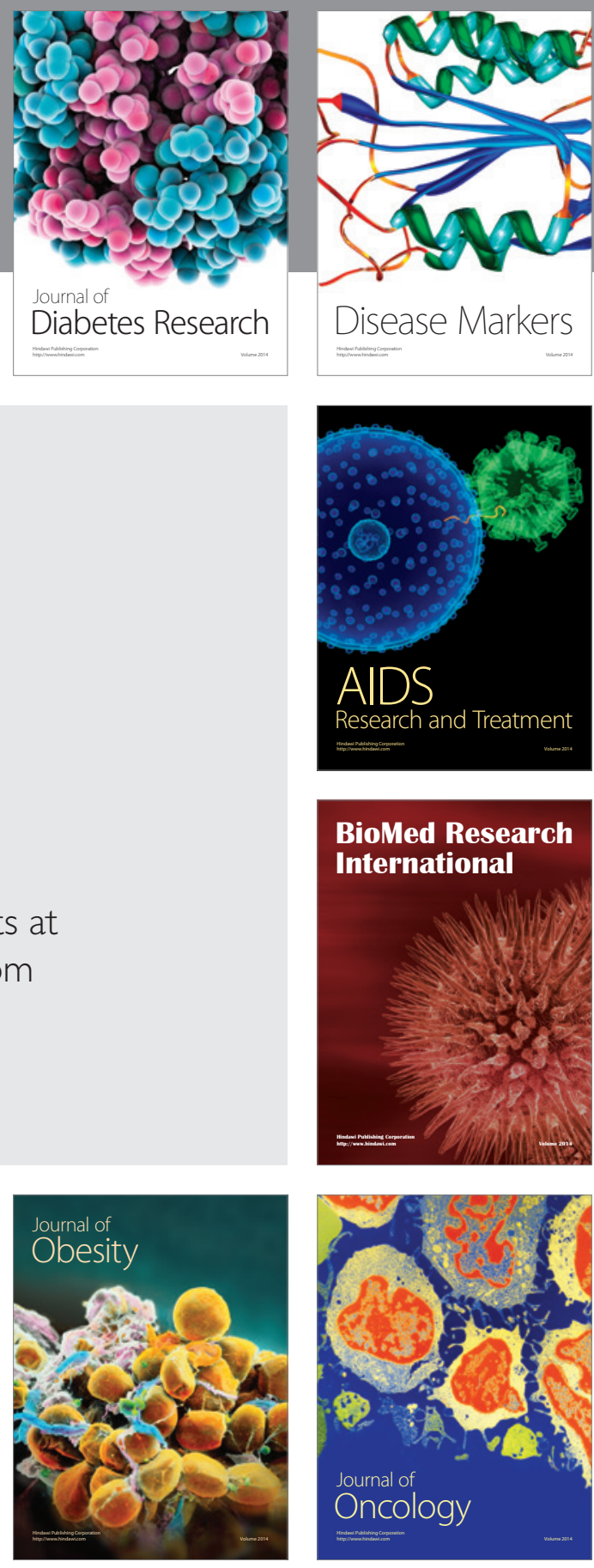

Disease Markers

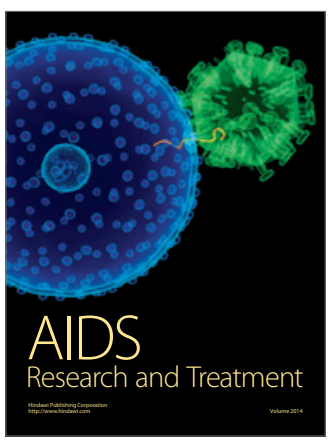

BioMed Research

International
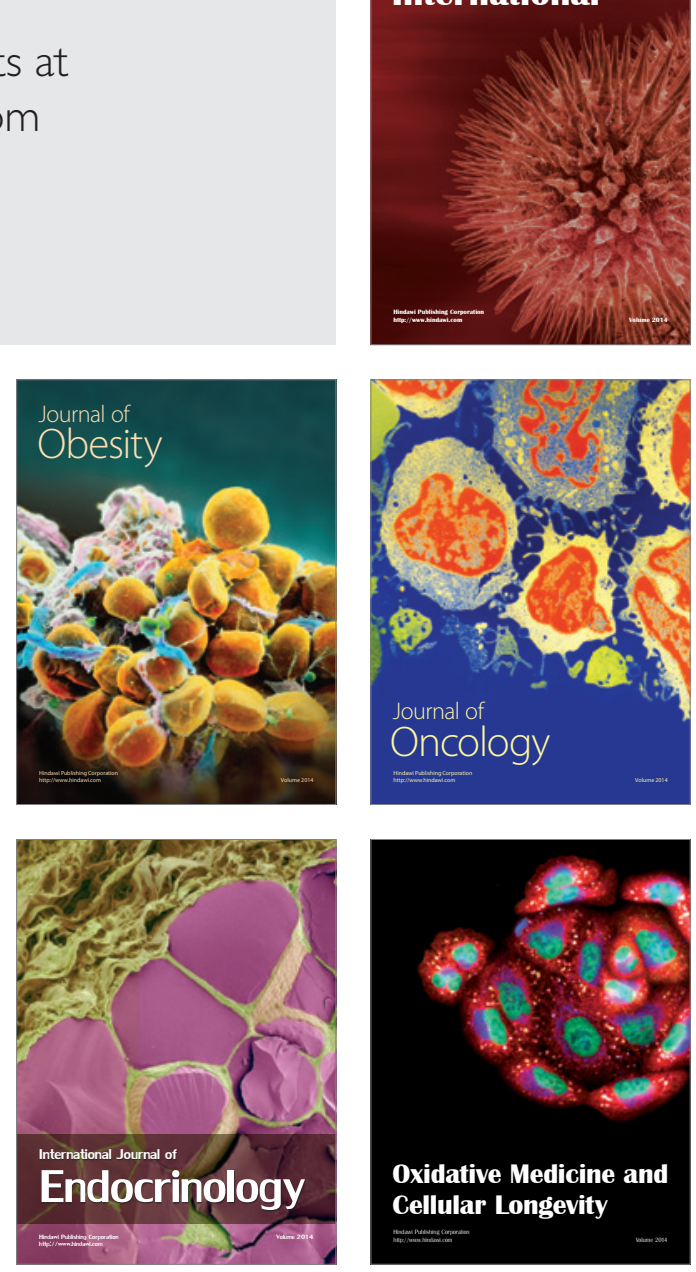